\title{
Editorial
}

\section{Precision cancer medicine: where to target?}

\author{
Qiang YU*, Jian DING* \\ Shanghai Institute of Materia Medica, Chinese Academy of Sciences, Shanghai 201203, China
}

Acta Pharmacologica Sinica advance online publication, 21 Sep 2015; doi: 10.1038/aps.2015.93

The discovery of the first oncogene $S r c$ in $1976^{[1]}$ opened up the field of molecular oncology and led to the discovery of oncogenes and anti-oncogenes that are involved in the formation and progression of cancers ${ }^{[2]}$. Nearly 4 decades of research on the functions and molecular mechanisms of oncogenes and anti-oncogenes and the related cell growth signaling pathways, has led to the understanding that cancer is characterized by uncontrolled cell growth that is regulated by multiple growth factors, growth factor receptors, cytoplasmic growth signal transducers and nuclear transcription factors, which collectively control nucleotides synthesis, DNA replication and cell division. Cancer treatments have been developed to target both the growth regulatory mechanisms as well as the DNA replication/cell division machinery. The traditional chemotherapies mostly target the cell division machinery, including nucleotide metabolism, DNA replication and cell division ${ }^{[3,4]}$. While such traditional chemotherapies are effective in killing cancer cells and blocking cancer cell growth, they suffer from the lack of targeting specificity as they are equally toxic to normal dividing cells, which are commonly present in the bone marrow, digestive tract and hair follicles. As a result, they result in severe side-effects such as myelosuppression, mucositis and hair loss. On the other hand, targeted cancer therapies, which are designed to attack the growth regulatory systems, particularly the genetically mutated and misbehaved specific cell growth pathway control molecules, have become increasingly popular in the past two decades. The targeted therapies have been proven to be more effective and less harmful to normal cells than the traditional cytotoxic or cytostatic chemotherapies, because the targeted molecules and their mutations are cancer cell-specific.

The promise of targeted cancer therapy relies on the discovery of key molecular mechanisms that are involved in regulating oncogenesis. Dozens of oncogenes and anti-oncogenes

\footnotetext{
* To whom correspondence should be addressed.

E-mail qyu@sibs.ac.cn (Qiang YU) Jding@simm.ac.cn (Jian DING)

Received 2015-09-08 Accepted 2015-09-14
}

have been identified along the growth signal transduction pathways, from growth factors, receptor tyrosine kinases, cytoplasmic signaling kinases to nuclear transcription factors $^{[5]}$. Key proteins in other growth-related pathways such as the cell death/survival pathways, protein degradation pathways are also found to play key roles in cancers ${ }^{[6-8]}$. Recently, immune cells and their regulatory pathways ${ }^{[9,10]}$, epigenetic modifications ${ }^{[11]}$, and non-coding $\mathrm{RNAs}^{[12]}$ were added to the growing list of cancer growth regulatory systems. The category of oncogenes and anti-oncogenes keeps expending. It is the discovery of the growth regulatory oncogenes and antioncogenes and the deep understanding of their functions and mechanisms that led to the discovery of a host of new generation anti-cancer drugs, which precisely target these oncogene and anti-oncogene products. Therefore, continuing discovery of genes and their products specifically function in promoting cancer cell growth is the basis of new anti-cancer drug development.

In this special issue of cancer research, we have invited a number of researchers who are actively involved in the discovery of growth regulatory molecules and anti-cancer drug development to write reviews on recent progresses and future directions of cancer drug discovery and cancer treatment, all of which aimed at developing novel cancer medicines to target precisely cancer cells. In this issue, Meng et al ${ }^{[13]}$ and Wang et $a l^{[14]}$ targeted one important family of kinases in cell growth control, the PI3K family, and described new inhibitors targeting distinct members of the PI3K family with more precision. Ge et $a l^{[15]}$ focused on tumor angiogenesis from a novel aspect of endogenous angiogenesis inhibitors and their therapeutic potentials in cancer treatment. Sun et al described the function and dysfunction of one of the key cancer-attacking cells in the immune system, the natural killer cells, in hepatocellular carcinomas and discussed their potential use in NK cell-based cancer immunotherapy ${ }^{[16]}$. Li et $a l^{[17]}$ and Le et al ${ }^{[18]}$ discussed microRNAs and alternative splicing respectively as potential new diagnostic biomarkers and targets for drug discovery. Finally, Sun et $a l^{[19]}$ reviewed recent findings in intra-tumor 
heterogeneity of cancer cells and their implications in guiding precision cancer treatment. All of these studies support the new exciting development in cancer medicine in order to tailor effective cancer treatment precisely based on the molecular signatures of cancers in target.

\section{References}

1 Stehelin D, Varmus HE, Bishop JM, Vogt PK. DNA related to the transforming gene(s) of avian sarcoma viruses is present in normal avian DNA. Nature 1976; 260: 170-3.

2 Hanahan D, Weinberg RA. Hallmarks of cancer: the next generation. Cell 2011; 144: 646-74.

3 Malhotra V, Perry MC. Classical chemotherapy: mechanisms, toxicities and the therapeutic window. Cancer Biol Ther 2003; 2(4 Suppl 1): S2-4.

4 Corrie PG. Cytotoxic chemotherapy: clinical aspects. Medicine 2011; 39: $717-22$.

5 Vogelstein B, Kinzler KW. Cancer genes and the pathways they control. Nat Med 2004; 10: 789-99.

6 Yuan TL, Cantley LC. PI3K pathway alterations in cancer: variations on a theme. Oncogene 2008; 27: 5497-510.

7 Kondo Y, Kanzawa T, Sawaya R, Kondo S. The role of autophagy in cancer development and response to therapy. Nat Rev Cancer 2005; 5: 726-34.

8 Jaattela M. Escaping cell death: survival proteins in cancer. Exp Cell Res 1999; 248: 30-43.

9 Elinav E, Nowarski R, Thaiss CA, Hu B, Jin C, Flavell RA. Inflammation- induced cancer: crosstalk between tumours, immune cells and microorganisms. Nat Rev Cancer 2013; 13: 759-71.

10 Grivennikov SI, Greten FR, Karin M. Immunity, inflammation, and cancer. Cell 2010; 140: 883-99.

11 Jones PA, Baylin SB. The fundamental role of epigenetic events in cancer. Nat Rev Genetics 2002; 3: 415-28.

12 Esteller M. Non-coding RNAs in human disease. Nat Rev Genetics 2011; 12: 861-74.

13 Meng LH, Zheng XF. Toward rapamycin analog (rapalog)-based precision cancer therapy. Acta Pharmacol Sin 2015; 36: 1163-9.

14 Wang X, Ding J, Meng LH. PI3K isoform-selective inhibitors: the next generation targeted cancer therapy. Acta Pharmacol Sin 2015; 36: 1170-6.

15 Ge RW, Rao N, Lee YF. Novel endogenous angiogenesis inhibitors and their therapeutic potential. Acta Pharmacol Sin 2015; 36: 1177-90.

16 Sun C, Sun HY, Xiao WH, Zhang C, Tian ZG. Natural killer cell dysfunction in hepatocellular carcinoma and NK cell-based immunotherapy. Acta Pharmacol Sin 2015; 36: 1191-9.

$17 \mathrm{Li} \mathrm{MH}$, Fu SB, Xiao HS. Genome-wide analysis of microRNA and mRNA expression signatures in cancer. Acta Pharmacol Sin 2015; 36: 1200-11.

18 Le KQ, Prabhakar BS, Hong WJ, Li LC. Alternative splicing as a biomarker and potential target for drug discovery. Acta Pharmacol Sin 2015; 36: 1212-8.

19 Sun XX, Yu Q. Intra-tumor heterogeneity of cancer cells and its implications in cancer treatment. Acta Pharmacol Sin 2015; 36: 1219-28. 